\title{
Acute Kidney Injury in patients referred for ECMO therapy
}

\author{
Anna Lima ${ }^{1}$, Sílvia Coelho ${ }^{2,3}$, Paulo Freitas ${ }^{2}$ \\ ${ }^{1}$ Nephrology Department, Hospital Prof Doutor Fernando Fonseca, Amadora, Portugal \\ 2 Intensive Care Department, Hospital Prof Doutor Fernando Fonseca, Amadora, Portugal \\ ${ }^{3}$ CEDOC - Chronic Diseases Research Center, Faculty of Health Sciences, University of Lisbon, Portugal
}

Dear editor,

Extracorporeal membrane oxygenation (ECMO) is a rescue therapy for critically ill patients with severe respiratory and/or circulatory failure, and its use has grown considerably in recent years. Patients submitted to ECMO are susceptible to acute kidney injury (AKI) due to reasons related to the primary disease but also to the ECMO treatment per se. ${ }^{1,2}$ The incidence of AKI is estimated at around $70-85 \%$ in patients undergoing ECMO and its presence greatly increases mortality rate. ${ }^{1,2}$

We aimed to evaluate the incidence, risk factors, and outcomes of patients with AKI transferred from the Intensive Care Unit (ICU) of Hospital Fernando Fonseca (HFF) to a reference center for ECMO treatment. We undertook a retrospective analysis of the electronic and paper records of patients referenced for ECMO from January 2011 to February 2018. Clinical, analytical and radiology data were collected, comprising not only the period of time from HFF admission to transfer to the ECMO reference center but also, when available, the time period from the return to the HFF post-ECMO, until hospital discharge. AKI was defined according to the Acute Kidney Injury Network (AKIN) criteria.

Our data comprised a total of 10 patients that were transferred from ICU to an ECMO center. The majority were male $(\mathrm{N}=8,80 \%)$ with a mean age of $51.4 \pm 6.9$ years. Pneumonia with respiratory insufficiency was the cause of hospital admission for all patients. $70 \%(\mathrm{~N}=7)$ of patients developed AKI before transference (14\% stage $3)$. SOFA score was higher in AKI vs non-AKI patients (median values 10 vs. 6 at HFF-ICU admission and 15 vs. 9 at transference to a tertiary center). Septic shock was present in all patients who developed AKI but in none of the non-AKI group. The AKI group also presented with more acidosis (median pH 7.32 vs. 7.39), higher carbon dioxide levels (mean pCO2 $53.6 \pm 17.1$ vs $45.5 \pm 4.45 \mathrm{mmHg}$ ) and lower PEEP parameters (mean $10.85 \pm 1.95$ vs $13.3 \pm 2.3 \mathrm{cmH}_{2} \mathrm{O}$ ). After transference to an ECMO center, 7 patients (70\%) were submitted to ECMO: 2 in the non-AKI group (28.57\%), 5 in the AKI group (71.43\%). ECMO duration was lower in the AKI group (mean $9 \pm 3.55$ vs. $11 \pm 5.65$ days). In the group of patients submitted to ECMO, 1 patient died (14.28\%, AKI group) and 2 patients (28.57\%) required renal replacement therapy (RRT). Nonetheless all the patients that survived $(n=9)$ recovered renal function ( 8 before and 1 after hospital discharge) and, until November 2018, none developed chronic kidney disease.
AKI was very common in patients referred to an ECMO center due to pneumonia with severe respiratory failure and was associated with higher severity disease. All survivals completely recovered renal function at one year. Although our study had a small sample, the results were similar to those described in the literature. Although AKI and RRT are associated with worse prognosis, they should not preclude ECMO referral. RRT initiation during ECMO therapy appears to be safe $^{3,4}$ but further studies are needed to access ideal timing for RRT in this population.

\section{$\underline{\text { Table } 1}$}

Characteristics of the study population according to the development of acute kidney injury; AKI lacute kidney injuryl

\begin{tabular}{l|c|c} 
& AKI (N=7) & NO AKI (N=3) \\
\hline SOFA at admition (median) & 10 & 6 \\
SOFA at transference (median) & 15 & 9 \\
Septic shock & $100 \%$ & $0 \%$ \\
Acidosis (median pH) & 7.32 & 7.39 \\
Carbon dioxide levels (mean pCO2) & $53.6 \pm 17.1$ & $45.5 \pm 4.45$ \\
PEEP parameters (mean) & $10.85 \pm 1.95$ & $13.3 \pm 2.3$ \\
ECMO therapy & $71.43 \%(\mathrm{~N}=5)$ & $28.57 \%(\mathrm{~N}=2)$ \\
ECMO duration (mean days) & $9 \pm 3.55$ & $11 \pm 5.65$ \\
Renal replacement therapy & $28.57 \%(\mathrm{~N}=2)$ & - \\
\hline
\end{tabular}

Disclosure of potential conflicts of interest: none declared

\section{References}

1. Chen YC, Tsai FC, Fang JT, Yang CW. Acute kidney injury in adults receiving extracorporeal membrane oxygenation. J Formos Med Assoc. 2014;113(11):778-785

2. Kilburn DJ, Shekar K, Fraser JF. The complex relationship of extracorporeal membrane oxygenation and acute kidney injury: causation or association? Biomed Res Int. 2016;2016:1094296.

3. Antonucci E, Lamanna I, Fagnoul D, Vincent JL, De Backer D, Silvio Taccone F. The impact of renal failure and renal replacement therapy on outcome during extracorporeal membrane oxygenation therapy. Artif Organs. 2016;40(8):746-754.

4. Husain-Syed F, Ricci Z, Brodie D, Vincent JL, Ranieri VM, Slutsky AS, et al. Extracorporeal organ support (ECOS) in critical illness and acute kidney injury: from native to artificial organ crosstalk. Intensive Care Med. 2018;44(9):1447-1459.

\section{Correspondence to:}

Anna Lima

Nephrology department, Hospital Prof. Dr Fernando Fonseca, Amadora, Portugal Email: anna.lima@hff.min-saude.pt 